\title{
Medical Image of the Week: Central Pontine Myelinolysis
}

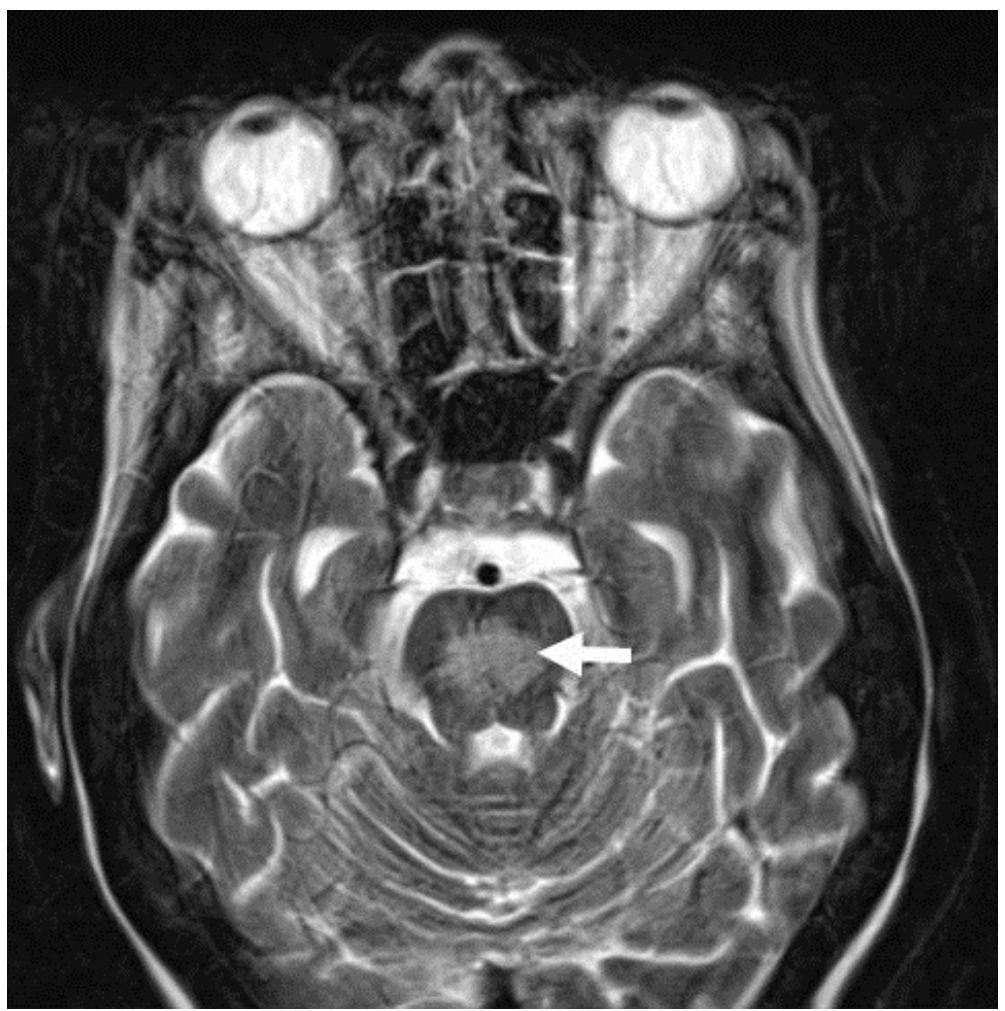

Figure 1. MRI axial T2 Image showing central pontine lesion consistent with osmotic demyelination.

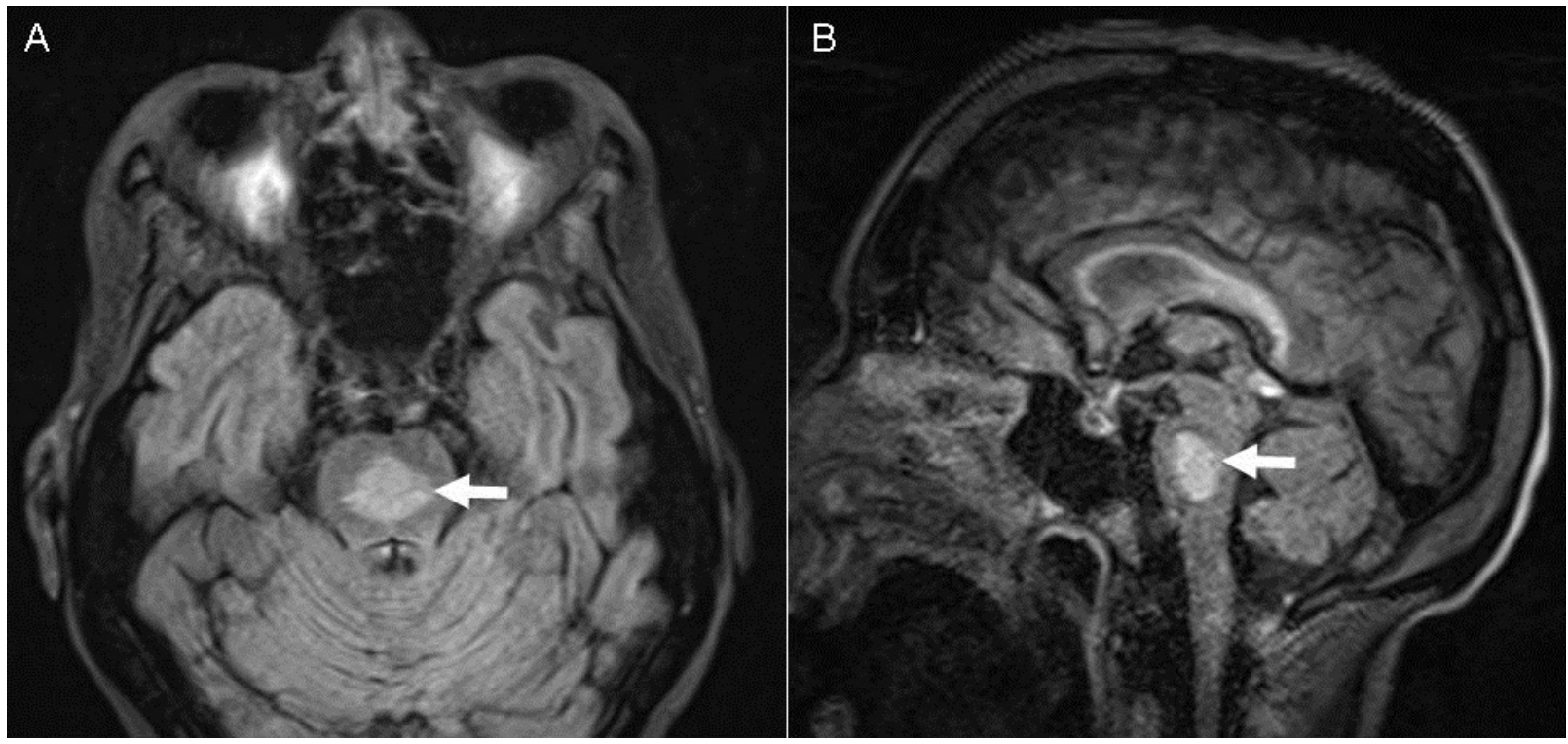

Figure 2. MRI axial (Panel A) and sagittal (Panel B) flair Images showing a hyperintensity located centrally within the pons with some sparing of the periphery measuring 1.6 by $1.3 \mathrm{~cm}$. 
A 38-year-old woman with history of alcohol abuse was admitted with generalized weakness, dehydration, alcoholic hepatitis, hyponatremia (serum sodium $116 \mathrm{mM} / \mathrm{L}$ ), and cachexia (BMI $19 \mathrm{~kg} / \mathrm{m} 2$ ). She developed hypoxemic respiratory failure after intravenous fluid resuscitation and required intubation and mechanical ventilation. Neurological exam revealed motor weakness, hyporeflexia, ataxia, and unsustained clonus. Neurology consultation was obtained and MRI revealed hyperintensity in the pons consistent with osmotic demyelination syndrome (1). Review of her records revealed her sodium level increased by $8 \mathrm{mM} / \mathrm{L}$ in first 6 hours of presentation, and then a slow increase of 4-6 mM/L daily to the normal range. She received nutritional support and aggressive physical therapy, and was discharged to skilled nursing facility after six weeks of hospitalization.

Tauseef Afaq Siddiqi, MD; Yeeck Sim, MD; Thi Nguyen, MD; Afshin Sam, MD

Division of Pulmonary, Allergy, Critical Care and Sleep Medicine, Department of Medicine University of Arizona

Tucson, AZ

\section{Reference}

Kumar S, Fowler M, Gonzalez-Toledo E, Jaffe SL. Central pontine myelinolysis, an update. Neurol Res. 2006;28(3):360-6. [CrossRef] [PubMed] 